\title{
Clinical Significance of Non-Mass-Like Enhancement of Preoperative Magnetic Resonance Imaging in Breast Cancer Considering Breast-Conserving Surgery
}

\author{
Min-Ji Park, Min-Young Park, Jin-Ok Kwon, Kyoung Sik Park, Yeong-Beom Yu, Jung-Hyun Yang, \\ Soo-Min Jung \\ Department of Surgery and Breast Cancer Center, Konkuk University Medical Center, Konkuk University School of Medicine, Seoul, Korea
}

Purpose: The purpose of this study was to investigate the significance of non-mass enhancement (NME) findings on preoperative breast magnetic resonance imaging (MRI) when invasive breast cancer patients with single lesions underwent breast-conserving surgery (BCS). Methods: We reviewed the preoperative MRI findings of 252 patients who underwent BCS from January 2014 to September 2016. Based on the MRI findings, we divided the patients into two groups, those who did and did not have NME, and we retrospectively analyzed the clinical outcomes of the two groups. Results: The NME group had 57 patients, and the no-NME group had 195 patients. The incidence of in situ lesions was higher in the NME group than in the no-NME group $(p<0.001)$. Additionally, the positive resection margin rate on frozen biopsy was higher in the NME group than in the no-NME group $(p=0.002)$. Conclusion: When preoperative MRI had NME findings, in situ lesions were more likely to accompany invasive breast cancer lesions, and the positive resection margin rate for frozen biopsy during BCS was high. Therefore, in these cases, the lesion should be excised more widely when BCS is performed, or frozen biopsy for resection margin during BCS should be performed if possible.

Key Words: Breast neoplasms, Magnetic resonance imaging, Segmental mastectomy

\section{INTRODUCTION}

The development of treatment modalities for breast cancer has greatly increased patients' survival rates. As a result, patients' quality of life has become more important than ever. Particularly regarding aesthetics, oncoplastic surgeries are being developed to preserve the breast and achieve superior cosmetic results [1]. However, regardless of the procedure, the most crucial aspect of breast-conserving surgery (BCS) is ensuring adequate safety margins. Therefore, preoperative radiologic findings play an essential role in planning these surgeries. In particular, breast cancer magnetic resonance imaging (MRI) findings play a crucial role in determining the scope and method of surgeries. Recent studies have demonstrated MRI specificity of $72 \%$ and sensitivity of $90 \%$ in breast cancer diagnosis [2]. In contrast, the sensitivity

\section{Correspondence: Soo-Min Jung}

Division of Breast and Endocrine Surgery, Department of Surgery, Konkuk University Medical Center, 120-1 Neungdong-ro, Gwangjin-gu, Seoul 05030, Korea

Tel: +82-2-2030-7589, Fax: +82-2-2030-7346, E-mail: lettoyam@naver.com Received: Jan 8, 2018 Revised: Feb 7, 2018 Accepted: Apr 14, 2018 of MRI in ductal carcinoma in situ (DCIS) patients varied from $77 \%$ to 96\% [3,4]. These results are clinically relevant for planning BCS based on MRI findings, especially when MRI shows non-mass enhancement (NME). According to the MR Breast Imaging Reporting and Data System (BI-RADS) classification system of the American College of Radiology, published in July 2003 [5] and recently updated in 2010, NME includes regional, segmental, ductal, and linear patterns. It has been reported that $57 \%$ of nonpalpable breast cancers show NME findings on MRI [6]. Although NME patterns may appear in diffuse benign lesions or inflammatory diseases [7], in actual operations, NME lesions are considered cancers and surgeons attempt to eliminate them entirely. However, localizing and resecting these lesions are often more difficult than expected. Therefore, we aimed to investigate the clinical significance of NME findings on MRI in patients considering BCS.

\section{METHODS}

We performed this study with the approval of our Institutional Re- 
view Board (KUH-1020074) following our institutional protocol. The informed consent was waived. In this study, only patients diagnosed with a single invasive lesion in pathologic results were included. In addition, to reduce any surgery-related bias, patients who underwent surgery by one experienced breast surgeon at our center from January 2014 to September 2016 were included. The exclusion criteria were as follows: (1) multiple invasive breast cancers on pathologic reports (even in the same quadrant or daughter nodule); (2) multifocal/multicentric breast cancer on image study (regardless of DCIS or invasive lesion): these lesions were excluded for a clear analysis of resection margins and NME; (3) bilateral breast cancer (for ease of analysis); (4) mastectomy patients; (5) history of ipsilateral breast operation (excision, excisional biopsy, and vacuum assisted breast biopsy), or (6) history of neoadjuvant chemotherapy. We retrospectively reviewed the medical records of 252 patients who underwent BCS and who had been diagnosed with a single invasive ductal carcinoma (IDC) with or without DCIS. We divided the 252 patients into two groups according to their NME findings on MRI: no-NME (a single mass lesion) or NME (a single NME lesion or a single mass lesion with NME) (Figure 1). We reviewed clinical findings, pathologic subtypes (classified as luminal A and B, based on Ki-67 < 14\% or $\geq 14 \%$ ), the presence of DCIS, and extensive intraductal component (EIC) for these patients. In this study, the expression for DCIS was defined as follows: (1) DCIS with invasive lesion: DCIS existing inside or outside the invasive lesion; (2) extra-tumoral DCIS: DCIS existing outside the invasive lesion, and (3) EIC: extra-tumoral DCIS occupying $\geq 25 \%$ of the breast cancer lesion. At our center, all patients underwent frozen biopsy for resection margin at the time of surgery. We also investigated the presence of cancer cells in frozen biopsy and final pathologic results for resection margin.

\section{MRI protocol of Konkuk Medical Center and image analysis}

The MRI examinations were performed using a 3.0-T MRI system (Signa HDxt; General Electric Medical Systems, Milwaukee, USA) with a dedicated 8-channel breast coil. After a transverse localizer image was obtained, sagittal fat-suppressed T2-weighted fast spin-echo images were obtained as well. Dynamic contrast-enhanced MRI examinations included one pre-contrast and five post-contrast sagittal image acquisitions using a fat-suppressed T1-weighted 3D fast-spoiled gradient-recalled echo sequence with parallel volume imaging. We also obtained delayed contrast-enhanced 3D fast-spoiled gradient-echo images with fat suppression in the axial plane. Gadoterate meglumine (Dotarem; Guerbet, Marans, France) was injected into the antecubital vein using an automated injector (Spectris Solaris; Medrad Europe, Maastricht, the Netherlands) at a dose of $0.1 \mathrm{mmol}$ per kilogram of body weight and a rate of $3 \mathrm{~mL} / \mathrm{sec}$ followed by a $20-\mathrm{mL}$ saline flush. Two breast imaging radiologists with seven and 11 years of experience interpreted the MRIs using the morphological MRI criteria described in the BI-RADS classification system.

\section{Statistical analysis}

We performed our statistical analyses using SPSS version 17.0 (SPSS Inc., Chicago, USA). Data are expressed either as frequencies or proportions. We tested for differences between groups using a chi-square test for qualitative variables and considered a $p$-value of $<0.05$ to be statistically significant.

\section{RESULTS}

Patient demographic data and pathologic report analysis results are
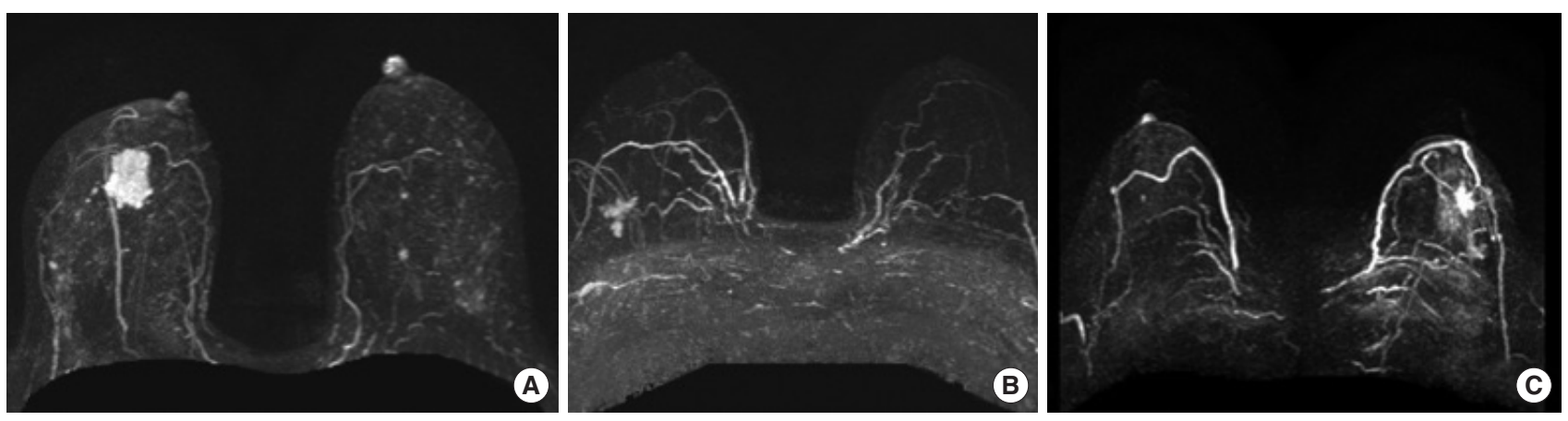

Figure 1. Magnetic resonance imaging. (A) Single mass lesion, (B) single non-mass-like lesion (NME), and (C) single mass with NME lesion. 
Table 1. Clinicopathological characteristics

\begin{tabular}{|c|c|c|c|}
\hline Variable & $\begin{array}{c}\text { No-NME group } \\
(n=195) \\
\text { No. }(\%)\end{array}$ & $\begin{array}{c}\text { NME group } \\
(n=57) \\
\text { No. }(\%)\end{array}$ & $p$-value \\
\hline Age $(y r)^{*}$ & $51.7 \pm 10.1$ & $50.2 \pm 9.4$ & 0.319 \\
\hline Site & & & 0.586 \\
\hline Left & $106(54.4)$ & $26(45.6)$ & \\
\hline Right & $89(45.6)$ & $31(54.4)$ & \\
\hline \multicolumn{4}{|l|}{ Tumor size } \\
\hline On MRI $(\mathrm{mm})^{*}$ & $19.6 \pm 8.8$ & $25.5 \pm 12.5$ & 0.001 \\
\hline On pathology $(\mathrm{mm})^{*}$ & $19.5 \pm 8.6$ & $20.6 \pm 12.3$ & 0.527 \\
\hline $\mathrm{T} 1$ & 117 & 27 & \\
\hline $\mathrm{T} 2$ & 78 & 29 & \\
\hline $\mathrm{T} 3$ & 0 & 1 & \\
\hline Axillary node metastasis & $44(22.6)$ & $22(38.6)$ & 0.025 \\
\hline $\mathrm{N} 1$ & 34 & 16 & \\
\hline N2 & 9 & 5 & \\
\hline N3 & 1 & 1 & \\
\hline Histologic grade & & & 0.813 \\
\hline 1 & $20(10.3)$ & $5(8.8)$ & \\
\hline 2 & $90(46.2)$ & $29(50.9)$ & \\
\hline 3 & 85 (43.6) & $23(40.4)$ & \\
\hline \multicolumn{4}{|l|}{ Hormonal receptor } \\
\hline $\mathrm{ER}(+)$ & $161(82.6)$ & $43(75.4)$ & 0.311 \\
\hline$(-)$ & $34(17.4)$ & $14(24.6)$ & \\
\hline $\mathrm{PR}(+)$ & $113(57.9)$ & $33(57.9)$ & 1.000 \\
\hline$(-)$ & $82(42.1)$ & $24(42.1)$ & \\
\hline HER2 & $(n=194)$ & $(n=56)$ & 0.047 \\
\hline$(+)$ & $32(16.5)$ & $17(30.4)$ & \\
\hline$(-)$ & $162(83.5)$ & $39(69.6)$ & \\
\hline $\mathrm{Ki}-67^{*}$ & $27.1 \pm 23.3$ & $25.6 \pm 22.3$ & 0.676 \\
\hline Molecular subtype & & & 0.008 \\
\hline Luminal A & $68(34.9)$ & $20(35.1)$ & \\
\hline Luminal B & $93(47.7)$ & $22(38.6)$ & \\
\hline HER2 type & $7(3.6)$ & $9(15.8)$ & \\
\hline TNBC & $27(13.8)$ & $6(10.5)$ & \\
\hline DCIS with invasive lesion & & & $<0.001$ \\
\hline$(+)$ & $142(72.8)$ & $54(94.7)$ & \\
\hline$(-)$ & $53(27.2)$ & $3(5.3)$ & \\
\hline Extra-tumoral DCIS & & & $<0.001$ \\
\hline$(+)$ & $83(42.6)$ & $50(87.7)$ & \\
\hline$(-)$ & $112(57.4)$ & $7(12.3)$ & \\
\hline EIC & & & $<0.001$ \\
\hline$(+)$ & $24(12.3)$ & $32(56.1)$ & \\
\hline$(-)$ & $165(84.6)$ & $23(40.4)$ & \\
\hline Missing data & $6(3.1)$ & $2(3.5)$ & \\
\hline Resection margin (frozen Bx.) & $(n=193)$ & & 0.002 \\
\hline Cancer(+) & $30(15.5)$ & $21(36.8)$ & \\
\hline$(-)$ & $163(84.5)$ & $36(63.2)$ & \\
\hline $\begin{array}{l}\text { Resection margin } \\
\text { (permanent Bx.) }\end{array}$ & & & 0.694 \\
\hline Cancer(+) & $12(6.2)$ & $5(8.8)$ & \\
\hline$(-)$ & $183(93.8)$ & $52(91.2)$ & \\
\hline
\end{tabular}

$\mathrm{NME}=$ non-mass-like enhancement; $\mathrm{MRI}=$ magnetic resonance imaging; $\mathrm{ER}=$ estrogen receptor; $\mathrm{PR}=$ progesterone receptor; $\mathrm{HER} 2$ = human epidermal growth factor receptor 2; TNBC= triple-negative breast cancer; DCIS= ductal carcinoma in situ; $\mathrm{EIC}=$ extensive intraductal component; $\mathrm{Bx}=$ biopsy. *Mean \pm SD. summarized in Table 1. The NME group had 57 patients, and the noNME group had 195; there were no statistically significant differences in age or breast cancer site between the two groups. Tumors measured on preoperative MRI were larger in the NME group ( $p=0.001)$. However, there was no difference in tumor size between the two groups measured from actual pathology reports: $19.5 \pm 8.6 \mathrm{~mm}$ in the noNME group and $20.6 \pm 12.3 \mathrm{~mm}$ in the NME group $(p=0.527)$. Axillary node metastasis was also significantly greater in the NME group (NME, 38.6\% vs. no-NME, 22.6\%; $p=0.025$ ). Histologic grade and hormone receptor status did not differ between the two groups when we analyzed the pathological results. There were more human epidermal growth factor receptor 2 (HER2)-positive patients in the NME group (29.8\%) than in the no-NME group $(16.4 \% ; p=0.047)$. In the molecular subtype analysis, HER2 type was found to be more abundant in the NME group $(p=0.008)$. The NME group contained more in situ lesions (94.7\%) than the no-NME group $(72.8 \% ; p<0.001)$. The proportion of extra-tumoral DCIS recorded on the pathology reports was significantly higher in the NME group (87.7\%) than in the noNME group $(42.6 \% ; p<0.001)$. In addition, the presence of EIC was significantly higher in the NME group than in the no-NME group (56.1\% vs. $12.3 \%$, respectively; $p<0.001)$. At our center, all patients underwent frozen biopsy for resection margin at the time of surgery. The positive resection margin rate of frozen biopsy was higher in the NME group than in the no-NME group (36.8\% vs. $15.5 \%$, respectively; $p=0.002$ ). The positive resection margin rates on permanent biopsy were $6.2 \%$ in the no-NME group and $8.8 \%$ in the NME group, but the difference was not significant $(p=0.694)$.

\section{DISCUSSION}

The efficacy of preoperative MRI for breast cancer patients has been verified by several studies, and MRI has become indispensable for planning surgery. In particular, for nonpalpable lesions, MRI provides a more accurate range of lesion size than ultrasound. According to a recent meta-analysis, preoperative MRI is associated with an increased odds of ipsilateral or contralateral prophylactic mastectomy as the surgical treatment for newly diagnosed breast cancer patients [8]. The reason for this result may be that MRI reveals lesions that are not usually found in breast ultrasound and mammography. Nonpalpable lesions or lesions not observed on mammography and ultrasonogra- 
phy are often observed as NME findings on MRI [5]. BI-RADS defines NME as non-mass enhancement of an area that may extend over small or large regions with internal enhancement characteristics that can be distinguished from the normal surrounding breast parenchyma. Several studies have reported that NME findings are often present in cases of histologically diagnosed DCIS [9,10]. Miyashita et al. [11] investigated MRI findings and final pathology results in patients diagnosed with DCIS through preoperative biopsy, and approximately $80 \%$ of the patients had NME findings; in $18 \%$ of those patients, final biopsy showed invasive lesions.

We conducted this study to determine whether the surgery range should be determined if there is NME on preoperative MRI from the viewpoint of the surgeon who performs the actual operation and to ascertain whether the cancer-positive rate in the actual resection margin is higher than when there are no NME findings. Kim et al. [12] studied the relationship between contrast enhancement lesions on dynamic contrast enhanced-MRI and the rates of re-excision and mastectomy conversion based on positive resection margins at the time of BCS. They reported that for $13.4 \%$ of patients, initially planned BCS was converted to mastectomy after MRI findings were confirmed, and re-excision was performed in $8.2 \%$ of patients after BCS. In addition, $22.2 \%$ of the patients with NME findings underwent re-excision, and the reoperation rate was high. In the present study, we only reviewed the postoperative pathology reports of MRI findings for patients with single-lesion IDCs. We found that when the NME findings were accompanied by a single mass lesion, or when there was only an NME lesion without a mass-like lesion, the postoperative positive resection margin rate at the time of surgery was more than twice as high as that of cases without NME findings (NME, 36.8\% vs. noNME, 15.4\%). However, there was no difference in the re-excision rate between the two groups because of the additional marginal resection through frozen biopsy during surgery. Another interesting finding of this study was that HER2 positivity was high when NME findings were present. To date, few studies have investigated the correlation between breast cancer imaging phenotype and molecular subtype. However, recent meta-analyses of HER2 overexpression and breast cancer imaging features have shown that irregular margins tended toward greater changes in HER2 overexpression (prevalence odds ratio, 1.56; 95\% confidence interval, 0.99-2.47; $p=0.06$; six studies) [13]. Other researchers have reported that the HER2-positive subtype of- ten shows microcalcifications $[14,15]$. However, no studies have examined the direct association of the HER2 subtype with NME, and more studies are required to determine the association of MRI findings with molecular subtypes. No convincing evidence has been found that preoperative MRI improves surgical outcomes, such as the rate of positive margins, re-excision, or breast conservation, in the average patient with breast cancer [16]. In conclusion, in the present study, we found that in the presence of NME findings on MRI, the rates of DCIS accompanied by invasive lesions and positive resection margins were high. Therefore, in these cases, to reduce re-excision rates, lesions should be resected more widely when BCS is performed, or frozen biopsy for resection margin during BCS should be performed if possible. In addition, it appears that the HER2 type (estrogen receptor-negative, progesterone receptor-negative) is more likely to accompany NME findings than other subtypes; therefore, breast MRI will be more helpful in determining the extent of resection.

\section{CONFLICT OF INTEREST}

The authors declare that they have no competing interests.

\section{REFERENCES}

1. Kim KD, Kim Z, Kuk JC, Jeong J, Choi KS, Hur SM, et al. Longterm results of oncoplastic breast surgery with latissimus dorsi flap reconstruction: a pilot study of the objective cosmetic results and patient reported outcome. Ann Surg Treat Res 2016;90:117-23.

2. Peters NH, Borel Rinkes IH, Zuithoff NP, Mali WP, Moons KG, Peeters PH. Meta-analysis of MR imaging in the diagnosis of breast lesions. Radiology 2008;246:116-24.

3. Menell JH, Morris EA, Dershaw DD, Abramson AF, Brogi E, Liberman L. Determination of the presence and extent of pure ductal carcinoma in situ by mammography and magnetic resonance imaging. Breast J 2005; 11:382-90.

4. Ballesio L, Di Pastena F, Gigli S, D’ambrosio I, Aceti A, Pontico M, et al. Non mass-like enhancement categories detected by breast MRI and histological findings. Eur Rev Med Pharmacol Sci 2014; 18:910-7.

5. Diebold T, Jacobi V, Krapfl E, von Minckwitz G, Solbach C, Ballenberger $\mathrm{S}$, et al. The role of stereotactic $11 \mathrm{G}$ vacuum biopsy for clari- 
fication of BI-RADS IV findings in mammography. Rofo 2003;175: 489-94.

6. Bartella L, Liberman L, Morris EA, Dershaw DD. Nonpalpable mammographically occult invasive breast cancers detected by MRI. AJR Am J Roentgenol 2006;186:865-70.

7. Thomassin-Naggara I, Salem C, Darai E, Bazot M, Uzan S, Marsault C, et al. Non-masslike enhancement on breast MRI: interpretation pearls. J Radiol 2009;90(3 Pt 1):269-75.

8. Houssami N, Turner RM, Morrow M. Meta-analysis of pre-operative magnetic resonance imaging (MRI) and surgical treatment for breast cancer. Breast Cancer Res Treat 2017;165:273-83.

9. Rosen EL, Smith-Foley SA, DeMartini WB, Eby PR, Peacock S, Lehman CD. BI-RADS MRI enhancement characteristics of ductal carcinoma in situ. Breast J 2007;13:545-50.

10. Jansen SA, Newstead GM, Abe H, Shimauchi A, Schmidt RA, Karczmar GS. Pure ductal carcinoma in situ: kinetic and morphologic MR characteristics compared with mammographic appearance and nuclear grade. Radiology 2007;245:684-91.

11. Miyashita M, Amano G, Ishida T, Tamaki K, Uchimura F, Ono T, et al. The clinical significance of breast MRI in the management of ductal carcinoma in situ diagnosed on needle biopsy. Jpn J Clin Oncol 2013;43:654-63.

12. Kim OH, Kim SJ, Lee JS. Enhancing patterns of breast cancer on preoperative dynamic contrast-enhanced magnetic resonance imaging and resection margin in breast conserving therapy. Breast Dis 2016;36:27-35.

13. Elias SG, Adams A, Wisner DJ, Esserman LJ, van't Veer LJ, Mali WP, et al. Imaging features of HER2 overexpression in breast cancer: a systematic review and meta-analysis. Cancer Epidemiol Biomarkers Prev 2014;23:1464-83.

14. Yamada T, Mori N, Watanabe M, Kimijima I, Okumoto T, Seiji K, et al. Radiologic-pathologic correlation of ductal carcinoma in situ. Radiographics 2010;30:1183-98.

15. Tse GM, Tan PH, Pang AL, Tang AP, Cheung HS. Calcification in breast lesions: pathologists' perspective. J Clin Pathol 2008;61:14551.

16. Houssami N, Turner R, Morrow M. Preoperative magnetic resonance imaging in breast cancer: meta-analysis of surgical outcomes. Ann Surg 2013;257:249-55. 